\title{
Quantum Information Splitting of Arbitrary Three-Qubit State by Using Seven-Qubit Entangled State
}

\author{
Dong-fen Li • Rui-jin Wang • Feng-li Zhang • \\ Fu-hu Deng
}

Received: 25 July 2014 / Accepted: 8 November 2014 / Published online: 22 November 2014 (C) The Author(s) 2014. This article is published with open access at Springerlink.com

\begin{abstract}
In this paper, we propose a scheme of quantum information splitting arbitrary three-qubit state by using seven-qubit entangled as quantum channel. The sender Alice first performs Bell-state measurements (BSMs) on her qubits pairs respectively and tells her measurement outcome to authorizers Bob to reconstruct the original state, then Charlie should carries out single-qubit measurement (SQM) on his qubits. According to the results from Alice and Charlie, Bob can reconstruct the original state by applying an appropriate unitary operation. After analyzing, the method achieved the desired effect of quantum information splitting (QIS). We also realize the QIS of arbitrary three-qubit state in cavity quantum electrodynamics (QED).
\end{abstract}

Keywords Quantum information splitting · Arbitrary three-qubit state · Quantum information · BSMs · QED

\section{Introduction}

The most striking features of quantum mechanics phenomenon is quantum entanglement [1-3]. It has some defects though the traditional cryptography is symmetrical password. It was difficult to solve a math problem based on mathematical cryptophytes. Its security is limited by the current computing power. Quantum entanglement is also one of the most important applications [4, 5], QIS is based on entanglement to achieve [6-20]. With the classical secret sharing of quantum information corresponding, the separation is based on the quantum theory. The current quantum secret sharing is a very meaningful topic, quantum information splitting can be described as follows: the use of entangled state as quantum

D. Li $(\bowtie) \cdot$ R. Wang $\cdot$ F. Zhang $\cdot$ F. Deng

School of Computer Science and Technology, University of Electronic Science and Technology of China, No.2006, XiYuan Ave, West Hi-Tech Zone, ChengDu, SiChuan, 611731,

People's Republic of China

e-mail: 201311060110@std.uestc.edu.cn

R. Wang

e-mail: ruijinwang@uestc.edu.cn 
channel connecting link between the separated information and receiver, quantum information splitting using quantum method for separating original quantum state information. At present, the research of quantum cryptography communication mainly includes quantum key distribution (QKD) [21-25]. Quantum cryptography communication is the art of transmitting the keys through a quantum channel between two parties we called Alice and Bob. Quantum secure direct communication (QSDC) [26-29], Quantum cryptography Shared, Quantum digital signature et al.

In our scheme, we describe a scheme to realize QIS of arbitrary three-qubit state by using seven-qubit entangled states as the quantum channel. Assume there are three legitimate parties, we say, Alice, Bob and Charlie. The sender of quantum information is Alice. Both of Bob and Charlie are agents. First of all, Alice carries out BSMs on her qubit pairs (A,1), $(\mathrm{B}, 3),(\mathrm{C}, 5)$, respectively. It is possible that Alice can get one of 64 kinds of measured outcomes with the basis of equality, then the surpluses particles will collapse into one of the 64 states $|\varphi i\rangle_{2467}(i=1,2, \ldots \ldots, 64)$. After measurement, Alice tells the BSMs outcomes to Bob and Charlie via a classical channel. The possibility of which Bob wants to reconstruct the original state depends on Charlie. If Charlie agrees Bob to reconstruct the original state information, he needs to carries out SQM on his qubit 7 and tells the measurement outcomes to Bob. According to the outcomes of Alice and Charlie, Bob can reconstruct the original state $|\Psi\rangle_{A B C}$ with an appropriate unitary transformation on his qubits.

The organization of this paper is as follows. In Section 2, we proposed a QIS protocol of arbitrary three-qubit state by using seven-qubit entangled state as quantum channel. In Section 3, we analysis the security against certain eavesdropping attacks. In Section 4, we completed the physical realization of the QIS in the cavity QED. In Section 5, the summary.

\section{Quantum Information Splitting of Arbitrary Three-Qubit State by Using Seven-Qubit Entangled State}

Suppose there are three legitimate parties, we say Alice, Bob and Charlie. Alice owner of the original information and also send the quantum information. The original unknown state can be written as

$$
\begin{aligned}
|\Psi\rangle_{A B C}= & a|000\rangle+b|001\rangle+c|010\rangle+d|011\rangle \\
& +e|100\rangle+f|101\rangle+g|110\rangle+h|111\rangle
\end{aligned}
$$

where $|a|^{2}+|b|^{2}+|c|^{2}+|d|^{2}+|e|^{2}+|f|^{2}+|g|^{2}+|h|^{2}=1$. Alice has sent the original state information to agents (Bob and Charlie). Bob is authorised to reconstruct the original state, he must cooperate with Charlie, otherwise he gets nothing. Alice needs to prepare a seven-qubit entangled state as quantum channel.

$$
\begin{aligned}
|\Psi\rangle_{1234567}= & \frac{1}{2 \sqrt{2}}(|000000\rangle+|0011000\rangle+|1100000\rangle-|1111000\rangle+|0000111\rangle \\
& +|0011111\rangle+|1100111\rangle-|1111111\rangle)
\end{aligned}
$$

The qubits A, B, C, 1, 3 and 5 belong to Alice, the qubits 2, 4 and 6 belongs to Bob, and the qubit 7 belong to Charlie, respectively. The whole system can be write as

$$
|\Omega\rangle=|\Psi\rangle_{A B C} \otimes|\Psi\rangle_{1234567}
$$

In order to achieve the QIS of arbitrary three-qubit state simply, Alice first carries out BSMs of his qubits pairs $(A, 1),(B, 3)$ and $(C, 5)$, respectively. She can get 64 kinds of 
possible measurement results with equal probability $\frac{1}{64}$. There are also 64 kinds of corresponding collapse states $|\varphi\rangle_{2467}^{i}(i=1,2, \ldots \ldots, 64)$ after the measurement.

$$
\begin{aligned}
& \left|\varphi^{1}\right\rangle_{2467}=\frac{1}{8}(a|0000\rangle \pm \pm b|0011\rangle++--c|0100\rangle \pm \mp d|0111\rangle \\
& ++++e|1000\rangle \pm \pm f|1011\rangle--++g|1100\rangle \mp \mp h|1111\rangle) \\
& \left|\varphi^{2}\right\rangle_{2467}=\frac{1}{8}(a|0011\rangle \pm \pm b|0000\rangle++--c|0111\rangle \pm \mp d|0100\rangle \\
& ++++e|1011\rangle \pm \pm f|1000\rangle--++g|1111\rangle \mp \pm h|1100\rangle) \\
& \left|\varphi^{3}\right\rangle_{2467}=\frac{1}{8}(a|0000\rangle \pm \pm b|0011\rangle++--c|0100\rangle \pm \mp d|0111\rangle \\
& ----e|1000\rangle \mp \mp f|1011\rangle++--g|1100\rangle \pm \mp h|1111\rangle) \\
& \left|\varphi^{4}\right\rangle_{2467}=\frac{1}{8}(a|0011\rangle \pm \pm b|0000\rangle++--c|0111\rangle \pm \mp d|0100\rangle \\
& ----e|1011\rangle \mp \mp f|1000\rangle++--g|1111\rangle \pm \mp h|1100\rangle) \\
& \left|\varphi^{5}\right\rangle_{2467}=\frac{1}{8}(a|0100\rangle \pm \pm b|0111\rangle++--c|0000\rangle \pm \mp d|0011\rangle \\
& ----e|1100\rangle \mp \mp f|1111\rangle++--g|1000\rangle \pm \mp h|1011\rangle) \\
& \left|\varphi^{6}\right\rangle_{2467}=\frac{1}{8}(a|0011\rangle \pm \pm b|0000\rangle++--c|0111\rangle \pm \mp d|0100\rangle \\
& ----e|1011\rangle \mp \mp f|1000\rangle++--g|1111\rangle \pm \mp h|1100\rangle) \\
& \left|\varphi^{7}\right\rangle_{2467}=\frac{1}{8}(a|0100\rangle \pm \pm b|0111\rangle++--c|0000\rangle \pm \mp d|0011\rangle \\
& ++++e|1100\rangle \pm \pm f|1111\rangle--++g|1000\rangle \mp \pm h|1011\rangle) \\
& \left|\varphi^{8}\right\rangle_{2467}=\frac{1}{8}(a|0011\rangle \pm \pm b|0000\rangle++--c|0111\rangle \pm \mp d|0100\rangle \\
& ++++e|1011\rangle \pm \pm f|1000\rangle--++g|1111\rangle \mp \pm h|1100\rangle) \\
& \left|\varphi^{9}\right\rangle_{2467}=\frac{1}{8}(----a|1100\rangle \mp \mp b|1111\rangle++--c|1000\rangle \pm \mp d|1011\rangle \\
& ++++e|0100\rangle \pm \pm f|0111\rangle++--g|0000\rangle \pm \mp h|0011\rangle) \\
& \left|\varphi^{10}\right\rangle_{2467}=\frac{1}{8}(----a|1111\rangle \mp \mp b|1100\rangle++--c|1011\rangle \pm \mp d|1000\rangle \\
& ++++e|0111\rangle \pm \pm f|0100\rangle++--g|0011\rangle \pm \mp h|0000\rangle) \\
& \left|\varphi^{11}\right\rangle_{2467}=\frac{1}{8}(----a|1100\rangle \mp \mp b|1111\rangle++--c|1000\rangle \pm \mp d|1011\rangle \\
& ---e|0100\rangle \mp \mp f|0111\rangle--++g|0000\rangle \mp \pm h|0011\rangle) \\
& \left|\varphi^{12}\right\rangle_{2467}=\frac{1}{8}(a|1000\rangle \pm \pm b|1011\rangle----c|1100\rangle \mp \mp d|1111\rangle++-- \\
& e|0000\rangle \pm \mp f|0011\rangle++--g|0100\rangle \pm \mp h|0111\rangle)
\end{aligned}
$$




$$
\begin{aligned}
\left|\varphi^{13}\right\rangle_{2467}= & \frac{1}{8}(a|1011\rangle \pm \pm b|1000\rangle---c|1100\rangle \mp \mp d|1100\rangle++-- \\
& e|0011\rangle \pm \mp f|0000\rangle++-g|0111\rangle \pm \mp h|0100\rangle) \\
\left|\varphi^{14}\right\rangle_{2467}= & \frac{1}{8}(a|0100\rangle \pm b|0111\rangle--c|0000\rangle \mp d|0011\rangle--e|1100\rangle \mp f|1111\rangle \\
& --g|1000\rangle \mp h|1011\rangle) \\
& --g|1011\rangle \mp h|1000\rangle) \\
\left|\varphi^{15}\right\rangle_{2467}= & \frac{1}{8}(a|0111\rangle \pm b|0100\rangle--c|0011\rangle \mp d|0000\rangle--e|1111\rangle \mp f|1100\rangle \\
& \\
\left|\varphi^{16}\right\rangle_{2467}= & \frac{1}{8}(a|1000\rangle \pm b|1011\rangle++c|1100\rangle \pm d|1111\rangle--e|0000\rangle \mp f|0011\rangle \\
& ++g|0100\rangle \pm h|0111\rangle) \\
& \frac{1}{8}(a|1011\rangle \pm b|1000\rangle++c|1111\rangle \pm d|1100\rangle--e|0011\rangle--f|0000\rangle \\
+ & +g|0111\rangle \pm h|0100\rangle)
\end{aligned}
$$

Then Alice tells the measurement outcome to the agents. The possibility of which Bob wants to reconstruct the original state depends on Charlie. If Charlie agrees Bob to reconstruct the original state information, he needs to carry out SQM on his qubit 7 in the basis of $\left\{| \pm\rangle_{7}\right\}$, where $| \pm\rangle=\frac{1}{\sqrt{2}}(|0\rangle \pm|1\rangle)$. According to the measurement outcomes of Alice and Charlie, Bob can reconstruct the original state $|\Psi\rangle_{A B C}$ with an appropriate unitary operation of his qubits 2, 4, 6 .

Bob can carry out an appropriate Pauli operator $\left(U_{1}, U_{2}, U_{3}, U_{4}\right)$ to reconstruct the original state $|\Psi\rangle_{A B C}$, where

$$
\begin{aligned}
U_{1}= & |000\rangle\langle 000|+| 001\rangle\langle 010\rangle+|010\rangle\langle 100|+| 011\rangle\langle 110| \\
& +|100\rangle\langle 001|-| 101\rangle\langle 011|-| 110\rangle\langle 101|+| 111\rangle\langle 111| \\
U_{2}= & |000\rangle\langle 000|+| 001\rangle\langle 010\rangle+|010\rangle\langle 100|+| 011\rangle\langle 110| \\
& -|100\rangle\langle 001|+| 101\rangle\langle 011|+| 110\rangle\langle 101|-| 111\rangle\langle 111| \\
U_{3}= & |000\rangle\langle 000|-| 001\rangle\langle 010\rangle-|010\rangle\langle 100|-| 011\rangle\langle 110| \\
& +|100\rangle\langle 001|+| 101\rangle\langle 011|-| 110\rangle\langle 101|-| 111\rangle\langle 111| \\
& \\
U_{4}= & |000\rangle\langle 000|-| 001\rangle\langle 010\rangle-|010\rangle\langle 100|-| 011\rangle\langle 110| \\
& -|100\rangle\langle 001|-| 101\rangle\langle 011|+| 110\rangle\langle 101|+| 111\rangle\langle 111|
\end{aligned}
$$

Charlie is required to measure his qubit 7 under the basis $| \pm\rangle=\frac{1}{\sqrt{2}}(|0\rangle \pm|1\rangle)$, then tells his measurement outcomes to Bob. According to Alice and Charlie's measurement outcomes, Bob can carries out an appropriate unitary operator to reconstruct the original state. 
Take, for example, $\left|\psi^{1}\right\rangle_{2467}$ to show the principle of this QIS protocol.

$$
\begin{gathered}
\left|\varphi^{1}\right\rangle=\frac{1}{8}(a|0000\rangle+b|0011\rangle+c|0100\rangle+d|0111\rangle e|1000\rangle+f|1011\rangle-g|1100\rangle \\
-h|1111\rangle) \\
=\frac{\sqrt{2}}{2}\left[|+\rangle_{7}(a|000\rangle+b|001\rangle+c|010\rangle+d|011\rangle+e|100\rangle+f|101\rangle\right. \\
\quad-g|110\rangle-h|111\rangle)_{246}+|-\rangle_{7}(a|000\rangle+b|001\rangle+c|010\rangle+d|011\rangle \\
\left.+e|100\rangle+f|101\rangle-g|110\rangle-h|111\rangle)_{246}\right]
\end{gathered}
$$

Charlie carries out SQM on qubit 7 and tells his measurement outcome to Bob.

If SQM result is $|+\rangle_{7}$, remaining qubits will collapse into $\left|\xi^{1}\right\rangle_{246}$ state.

$$
\left|\xi^{1}\right\rangle_{246}=(a|000\rangle+b|001\rangle+c|010\rangle+d|011\rangle+e|100\rangle+f|101\rangle-g|110\rangle-h|111\rangle)
$$

If SQM result is $|-\rangle_{7}$, remaining qubits will collapse into $\left|\xi^{2}\right\rangle_{246}$ state.

$$
\left|\xi^{2}\right\rangle_{246}=(a|000\rangle+b|001\rangle+c|010\rangle+d|011\rangle+e|100\rangle-f|101\rangle-g|110\rangle+h|111\rangle)
$$

In order to reconstruct the original state information, Bob must carry out a corresponding single qubit unitary operation $U_{1}$ or $U_{3}$ on the qubit 3 .

$$
a|000\rangle+b|001\rangle+c|010\rangle+d|011\rangle e|100\rangle-f|101\rangle-g|110\rangle+h|111\rangle
$$

After Bob get Alice and Charlie's measurement outcomes, he needs to carry out an appropriate unitary operator to reconstruct the original state.

\section{Security Analyses}

Suppose that an eavesdropper Eve, tries to attack the information between Alice, Bob and Charlie. Eve obtains original state information through measure the entanglement auxiliary qubit. If Alice, Bob and Charlie are not aware of the eavesdropper Eve, then after Alice performs three Joint BSMs, remaining qubits of Bob, Charlie and Eve will collapses into a five-qubit entangled state. Charlie performs a single qubit measurement of his qubit 7 , the Bob-Eve system will collapse into a product state, leaving Eve with no information about original state.

Suppose Alice gets the result $\left|\Phi^{+}\right\rangle_{A 1}\left|\Phi^{+}\right\rangle_{B 3}\left|\Phi^{+}\right\rangle_{C 5}$, then the combined state of Bob, Charlie and Eve would be

$$
\begin{aligned}
|\Theta\rangle_{2467}=\frac{1}{8 \sqrt{2}} & (a|00000\rangle+a|00001\rangle+b|00110\rangle+b|00111\rangle \\
& +c|01000\rangle+c|01001\rangle+d|01110\rangle+d|01111\rangle \\
& +e|10000\rangle+e|10001\rangle+f|10110\rangle+f|10111\rangle \\
& -g|11000\rangle-g|11001\rangle-h|11110\rangle-h|11111\rangle)
\end{aligned}
$$


If Charlie gets the state $\{|+\rangle\}_{7}$, then Bob-Eve system will collapse into $\left|\zeta^{1}\right\rangle_{246 E}$ state; If Charlie obtains the state $\{|-\rangle\}_{7}$, then Bob-Eve system will collapse into $\left|\zeta^{2}\right\rangle_{246 E}$ state.

$$
\begin{aligned}
\left|\zeta^{1}\right\rangle_{246 E}= & (a|0000\rangle+a|0001\rangle+b|0010\rangle+b|0011\rangle+c|0100\rangle+c|0101\rangle \\
& +d|0110\rangle+d|0111\rangle+e|1000\rangle+e|1001\rangle+f|1010\rangle+f|1011\rangle \\
& -g|1100\rangle-g|1101\rangle-h|1110\rangle-h|1111\rangle) \\
= & (a|000\rangle+b|001\rangle+c|010\rangle+d|011\rangle+e|100\rangle+f|101\rangle-g|110\rangle \\
& \quad-h|111\rangle_{246} \frac{1}{\sqrt{2}}(|0\rangle+|1\rangle)_{E} \\
& \quad+d|0110\rangle+d|0111\rangle+e|1000\rangle+e|1001\rangle-f|1010\rangle \\
& \quad-f|1011\rangle-g|1100\rangle-g|1101\rangle+h|1110\rangle+h|1111\rangle) \\
\left|\zeta^{2}\right\rangle_{246 E}= & (a|0000\rangle+a|0001\rangle+b|0010\rangle+b|0011\rangle+c|0100\rangle+c|0101\rangle \\
& +h|111\rangle_{246} \frac{1}{\sqrt{2}}(|0\rangle+|1\rangle)_{E}
\end{aligned}
$$

Obviously, Eve has no chance to get the original qubits state.

\section{Physical Realizations}

QED is considered to be one of the idea system. In recent years, QIS attracts great interest in cavity QED. Polyatom has been used to carry out transportation in cavity QED [30-33]. An arbitrary $\mathrm{N}$-qubit state in cavity QED system phenomenon more and more arouses the interest. It is well known that the entangled state is maximally connected and persistency than the GHZ-state. In addition, the entangled states are robust against decoherence [34, 35]. We also consider that $\mathrm{N}$ identical two two-level atoms in a single-mode cavity with a strong classical driving field [36]. We drive the two ions with a laser beam at the same time. Suppose that the cavity field is initially in the vacuum state. In rotating-wave approximation, the Hamiltonian (we set $\hbar=1$ ) describes such a system and it can be written as

$$
\begin{array}{r}
H=v a^{\dagger} a+\omega_{0} \sum_{j=m, n} \sigma_{z, j}+\Omega \sum_{j=m, n}\left[e^{-i\left[\left(\omega_{0}-v-\delta\right) t-\eta\left(a+a^{\dagger}\right)+\phi\right]}\right. \\
\left.+\sigma_{j}^{+}+\text {H.c. }\right]
\end{array}
$$

where $\sigma_{j}^{+}=\left|1_{j}\right\rangle\left\langle 0_{j}\left|, \sigma_{j}^{-}=\right| 0_{j}\right\rangle\left\langle 1_{j}\right|$ and $\sigma_{z, j}=\frac{1}{2}\left(\left|1_{j}\right\rangle\left\langle 1_{j}|-| 0_{j}\right\rangle\left\langle 0_{j}\right|\right)$ with $\left|0_{j}\right\rangle$ is the ground states, $\left|1_{j}\right\rangle$ is the excited states of the $\mathrm{j}$-th atom. The subscripts $m$ and $n$ expresses two different ions, $\omega_{0}$ expresses atomic transition frequency, $v$ express the collective motion of the ions frequency. Under certain condition of $\delta \leq v$, the stretch modes of the excitation can be neglected. $a$ express annihilation operator for the cavity mode, $a^{\dagger}$ expresses creation operator for the cavity mode. $\Omega$ expresses the Rabi frequency, $\phi$ expresses the phase of the laser vector. $\eta$ is a Lamb-Dicke parameter with the mass $M$ of an ion under the condition of the $\eta$, where $\eta=\frac{k}{\sqrt{2 v M}}, k$ expresses the wave vector. Under the conditions of $\delta \geq$ 
$\eta \Omega, \phi=\frac{\pi}{2}$, the vibration frequency $v$ is much larger than the other frequencies. Under rotating-wave approximation, the effective Hamiltonian can be written as

$$
H_{e}=\lambda\left[\frac{1}{2} \sum_{j=m, n}\left(\left|0_{j}\right\rangle\left\langle 0_{j}\right|\right)+\left(\left|1_{j}\right\rangle\left\langle 1_{j}\right|\right)+\left(S_{m}^{+} S_{n}^{+}+S_{m}^{+} S_{n}^{-}+H . c .\right]\right.
$$

where $\lambda=\frac{2(\Omega \eta)^{2}}{\delta}$. The Hamiltonian is one of the cavity field state of which permitting it to be in a thermal state. The two-ion system's evolution operator is given by

$$
U(t)=e^{-i H_{e} t}
$$

Alice wants to split an arbitrary three-qubit state in cavity QED system, she must carry out two joint BSMs. We execute the rotation $|1\rangle \rightarrow i|1\rangle$ on ion $i$, we can get outcomes as follows

$$
\begin{aligned}
\left|\Phi^{+}\right\rangle_{i j} \rightarrow \frac{1}{\sqrt{2}}(|00\rangle+i|11\rangle)_{i j} & \left|\Phi^{-}\right\rangle_{i j} \rightarrow \frac{1}{\sqrt{2}}(|00\rangle-i|11\rangle)_{i j} \\
\left|\Psi^{+}\right\rangle_{i j} \rightarrow \frac{1}{\sqrt{2}}(|01\rangle+i|10\rangle)_{i j} & \left|\Psi^{-}\right\rangle_{i j} \rightarrow \frac{1}{\sqrt{2}}(|01\rangle-i|10\rangle)_{i j}
\end{aligned}
$$

We let atoms $i$ and $j$ interact at the same time with a single-mode cavity and by a strong classical field under the conduct. Picking the interaction time and the Rabi frequency appropriately where $\lambda t=\frac{\pi}{4}$. We can get the following evolution states

$$
\begin{aligned}
\left|\Phi^{+}\right\rangle_{i j} \rightarrow|00\rangle_{i j} & \left|\Phi^{-}\right\rangle_{i j} \rightarrow-i|11\rangle_{i j} \\
\left|\Psi^{+}\right\rangle_{i j} \rightarrow|01\rangle_{i j} & \left|\Psi^{-}\right\rangle_{i j} \rightarrow-i|10\rangle_{i j}
\end{aligned}
$$

The joint BSMs can be completed by gauging ions $i$ and $j$ separately if we have discarded the common phase factor $e^{-i \pi / 4}$. After we finished the operation, Bob can successfully reconstruct the original three-qubit state $|\Psi\rangle_{A B C}$. So, QIS of arbitrary three-qubit state in cavity QED is also implemented.

\section{Conclusion}

In summary, we have supposed a seven-qubit entangled state via arbitrary three-qubit quantum information splitting. In our scheme, Alice first performs two BSMs and tells her measurement outcomes to Bob and Charlie. Bob is authorized to reconstruct the original state, he must cooperate with Charlie, otherwise he gets nothing. Charlie needs to carry out a SQM on his qubit and tells Bob the measurement outcomes. According to measurement outcomes from Alice and Charlie, Bob can reconstruct the original state with an appropriate unitary operation of his qubits. This protocol is considered to be secure. This scheme is experimentally achievable and also can be used to construct a controlled quantum channel that is useful in the future quantum information computation. We also realize QIS of arbitrary three-qubit state in cavity QED.

Open Access This article is distributed under the terms of the Creative Commons Attribution License which permits any use, distribution, and reproduction in any medium, provided the original author(s) and the source are credited. 


\section{References}

1. Bennett, C.H., Brassard, G.: Proc: IEEE Int. Conf. Comput. Syst. Signal Processing, p. 175. IEEE, New York (1984)

2. Ekert, A.K.: Phys. Rev. Lett. 67, 661 (1991)

3. Bennett, C.H., Brassard, G., Mermin, N.D.: Phys. Rev. Lett. 68, 557 (1992)

4. Deng, F.G., Long, L.: Phys. Rev. A 68, 042315 (2003)

5. Li, X.H., Deng, F.U., Zhou, H.Y.: Phys. Rev. A 78, 022321 (2008)

6. Li, J., Song, D.J., Guo, X.J., Jin, G.B.: Chin. Phys. C 36, 31 (2012)

7. Huang, W., Wen, Q.Y., Jia, H.Y., Qin, S.J., Gao, F.: Chin. Phys. B 21, 100308 (2012)

8. Sun, Z.W., Du, R.G., Long, D.Y.: Int. J. Theor. Phys. 51, 1946 (2012)

9. Gu, B., Zhang, C.Y., Cheng, G.S., Huang, Y.G.: China Phys. B 54, 942 (2011)

10. Ren, B.C., Wei, H.R., Hua, M., Li, T., Deng, F.G.: Eur. Phys. J. D 67, 30 (2013)

11. Long, G.L., Liu, X.S.: Phys. Rev. A 65, 032302 (2012)

12. Deng, F.G., Long, G.L., Liu, X.S.: Phys. Rev. A 68, 042317 (2003)

13. Deng, F.G., Long, G.L.Z.: Phys. Rev. A 69, 052319 (2004)

14. Wang, C., Deng, F.G., Li, Y.S., Liu, X.S., Long, G.L.: Phys. Rev. A 71, 044305 (2005)

15. Wang, C., Deng, F.G., Long, G.L.: Opt. Commun. 253, 15 (2005)

16. Zhu, A.D., Xia, Y., Fan, Q.B., Zhang, S.: Phys. Rev. A 73, 022338 (2006)

17. Gao, F., Guo, F.Z., Wen, Q.Y., Zhu, F.C.: Phys. Mech. Astron. 51, 1853 (2009)

18. Wang, T.J., Li, T., Du, F.F., Deng, F.G.: Chin. Phys. Lett. 28, 040305 (2011)

19. Liu, D., Chen, J.L., Jiang, W.: Int. J. Theor. Phys. 51, 2923 (2012)

20. Bennet, C.H., Brassard, G., Crepeau, C.: Phys. Rev. Lett. 70, 1895-1899 (1993)

21. Karlsson, A., Bourennane, M.: Phys. Rev. A 58, 4394 (1998)

22. Gorbachev, V.N., Trubilko, A.I., Rodichkina, A.A.: Phys. Lett. A 314, 267 (2003)

23. Agrawal, P., Pati, A.: Phys. Rev. A 74, 062320 (2006)

24. Nie, Y.Y., Hong, Z.H., Hong, Y.B., Yi, X.J., Li, S.S.: Theor. Phys. 48, 1485 (2009)

25. Bouwmeester, D.: Nature 390, 575-579 (1997)

26. Walther, P., Resch, K.J., Rudolph, T., Schenck, E., Weinfurter, H., Vedral, V., Aspelmeyer, M., Zeilinger, A.: Nature 434, 169-176 (2005)

27. Sreraman, M., Prasanta, K.P.: Phys. Rev. A 78, 062333 (2008)

28. Li, Y.H., Liu, J.C., Nie, Y.Y.: Commun. Theor. Phys. 55, 421-425 (2011)

29. Ralidharan, S., Panigrahi, P.K.: Phys. Rev. A 78(6), 062333 (2003)

30. Deng, F.G., Li, C.Y., Li, Y.S., et al.: Phys. Rev. A 59, 1829-1834 (1999)

31. Hillery, M., Buzek, V., Berthiaume, A.: Phys. Rev. A 59, 1829-1834 (2010)

32. Li, Y.H., Nie, Y.Y.: Commun. Theor. Phys. 57, 995-998 (2012)

33. Li, Y.H., Nie, L.P.: Int. J. Theor. Phys. 52, 961-967 (2013)

34. Zhang, W., Xiong, K.W., Zuo, X.Q., Zhang, Z.Y.: Commun. Theor. Phys. 59, 157-164 (2013)

35. Nie, Y.Y., Li, Y.H., Wang, Z.S.: Quantum Inf. Process 12, 437-448 (2013)

36. Tang, M.L., Zhu, H.P.: Int. J. Theor. Phys. 52, 2686-2691 (2013) 